\title{
IUFOST2006/829 Bone health-supporting activity of carotenoids
}

\author{
J. Levy \\ Ben-Gurion University, Clinical Biochemistry, Faculty of Health Sciences, PO Box 653, 84105 Beer-Sheva, \\ Israel \\ lyossi@bgu.ac.il
}

\begin{abstract}
A diet rich in fruit and vegetables has been linked to bone health and prevention of osteoporosis, a common degenerative bone disease. In particular, recent studies suggest a role for carotenoids in bone health; but evidence is scarce. Here we present results which support the role of carotenoids such as lycopene, betacarotene, phytoene and astaxanthin in bone health. It is well established that bone health is the result of two opposing processes, bone formation and bone resorption. Bone formation is governed by osteoblast cells while bone resorption is governed by osteoclast cells. These opposing processes are regulated by several hormones including vitamin D (bone formation) and glucorticoids (bone reasorption). To evaluate the role of dietary carotenoids in bone health, we analyzed their effects on the expression of bone-forming proteins such as alkaline phosphatase (ALP), osteoprotegerin (OPG) and Vitamin D receptors in human osteoblast-like cells. We found that all the tested carotenoids increased the level of these bone-building proteins. Moreover, the carotenoids augmented the activity of vitamin D which is known to increase the levels of bone supporting proteins. These results suggest that dietary carotenoids may work in combination with vitamin D to improve bone health. In addition we examined the carotenoid effects on bone resorption activity of glucocorticoids which is a major side effect of therapy with these hormones. Interestingly we found that lycopene and other tomato carotenoids prevented the glucocorticoid-induced decrease of OPG levels suggesting that carotenoids may prevent bone resorption by inhibiting glucocorticoid activity. To determine the molecular mechanisms underlying carotenoid action we focused on transcription, one of the cellular mechanisms responsible for the modulation of protein levels. We found that carotenoids increase the activity of the antioxidant response element transcription systems. Interestingly, activation of this transcription system is mediating the effect of carotenoids and other active dietary ingredient in bone formation and resorption.
\end{abstract}

